\title{
Addendum to: Determination of the electric field gradient and the magnetic field in Mössbauer spectroscopy ${ }^{\star}$
}

Eur. Phys. J. B (2018) 91: 292, https://doi.org/10.1140/epjb/e2018-90379-x

Krzysztof R. Szymański ${ }^{a}$

University of Białystok, Faculty of Physics, Ciołkowskiego 1L, 15-245 Białystok, Poland

Received 28 June 2019

Published online 12 August 2019

(C) EDP Sciences / Società Italiana di Fisica / Springer-Verlag GmbH Germany, part of Springer Nature, 2019

Acknowledgments were missing in the published paper. This erratum provides the acknowledgments:

This work was partly supported by the National Science Centre under grant OPUS no 2018/31/B/ST3/00279.

\footnotetext{
${ }^{\star}$ The online version of the original paper can be found at https://doi.org/10.1140/epjb/e2018-90379-x

${ }^{a}$ e-mail: k.szymanski@uwb.edu.pl
} 